\title{
The covariance of chiral fermions theory
}

\author{
K. Andrzejewski, ${ }^{a}$ Y. Brihaye, ${ }^{b}$ C. Gonera, ${ }^{a}$ J. Gonera, ${ }^{a}$ P. Kosiński ${ }^{a, 1}$ and \\ P. Maślanka ${ }^{a}$
}

${ }^{a}$ Department of Computer Science, Faculty of Physics and Applied Informatics, University of Lodz, Lodz, Poland

${ }^{b}$ Physique-Mathématique, Université de Mons-Hainaut, Mons, Belgium

E-mail: krzysztof .andrzejewski@uni.lodz.pl, Yves.BRIHAYE@umons.ac.be, cezary.gonera@uni.lodz.pl, joanna.gonera@uni.lodz.pl, piotr.kosinski@uni.lodz.pl, pawel.maslanka@uni.lodz.pl

ABSTRACT: The quasiclassical theory of massless chiral fermions is considered. The effective action is derived using time-dependent variational principle which provides a clear interpretation of relevant canonical variables. As a result their transformation properties under the action of Lorentz group are derived from first principles.

KeYwords: Space-Time Symmetries, Gauge Symmetry

ARXIV EPRINT: 1801.09447

\footnotetext{
${ }^{1}$ Corresponding author.
} 


\section{Contents}

1 Introduction 1

2 Free Weyl fermions $\quad 3$

$\begin{array}{llr}3 & \text { Interaction with electromagnetic field } & 8\end{array}$

$\begin{array}{lll}4 & \text { Conclusions } & 10\end{array}$

$\begin{array}{ll}\text { A Variation of action to the first order in } \hbar & 10\end{array}$

\section{Introduction}

In recent years a renewed interest is observed concerning chiral anomalies, their physical consequences (in particular, for kinetic theory) and relations to topology and Berry curvature [1]-[25]. For many purposes (e.g. the dynamics in weak and slowly varying external fields) one can rely on semiclassical approximation. In particular, within this approximation the Weyl equation for helicity $-\frac{1}{2}$ massless charged fermions is replaced by the semiclassical dynamics summarized in the action functional

$$
I=\int\left((\vec{q}+\vec{A}) \cdot d \vec{x}-\left(\varepsilon+A^{0}\right) d t+\vec{\alpha}(\vec{q}) \cdot d \vec{q}\right),
$$

where $\vec{q}$ denotes gauge-invariant (kinetic) momentum, $\vec{\alpha}(\vec{q})$ is the vector potential describing the Berry monopole in momentum space while

$$
\varepsilon=|\vec{q}|+\frac{\vec{q} \cdot \vec{B}}{2|\vec{q}|^{2}}
$$

Eq. (1.1) can be derived from Weyl Hamiltonian by making the semiclassical approximations to path-integral representation of transition amplitude [18]. The obvious trouble with eq. (1.1) is that it lacks manifest Lorentz symmetry (in the absence of external fields) or Lorentz covariance, in spite of the fact that it is derived from covariant Weyl equation. In ref. [18] a modified transformation rule under Lorentz boosts has been proposed for the particle dynamical variables, involving $O(\hbar)$ corrections, which leaves invariant the dynamics described by the action (1.1). It reduces to the standard Lorentz form for spinless particles. The modified rules involve helicity dependent terms and close only on-shell.

The resulting situation attracted much attention [14-17, 22-24]. In particular, Duval et al. were able to derive the modified transformation rules for chiral fermions from Soriau model [26] of massless spinning particles by applying the procedure called spin enslaving. Then similar results have been obtained by using the coadjoint orbits method [25]. 
The present paper is devoted to the further study of the covariance problem for chiral fermions. We consider the latter to be important because it can shed more light on the question of localizability and the meaning of position operator in relativistic quantum theory, in particular in the case of massless particles. In a very nice paper [27] Skagerstam noticed that the existence of position operator with standard transformation properties and commutation rules would imply that the massless irreducible representation of Poincare group accommodates all $2 \lambda+1$ chiralities, which is not the case (note that already Newton and Wigner [28] found that the position operator with commuting coordinates exists only for $\lambda=0, \frac{1}{2}$; in the latter case both helicities should be considered). In fact, the results obtained in [25] imply that we have the following choice: either (i) the coordinate operator has noncommuting components or (ii) it transforms nonlinearly under rotations. As a result the transformation properties of coordinates of massless particles with nonzero helicity are exotic which is nicely intuitively explained in [18] (see also last refs. [14-17]). This reasoning, when applied to photons, leads to the so-called relativistic Hall effect of light [29]-[33-35]. The problem has, however, more general context. It is well known $[36,37]$ that, due to the fact that in the quantum relativistic regime the presence of interactions excludes particle number conservation, the notion of localizability has a restricted meaning. Obviously, as long as noninteracting theory is considered we do not have to bother about localizability. Everything can be formulated in momentum space which provides a natural framework for Poincare covariance. One can, of course, also play with enveloping algebra of Poincare one to propose and construct various types of position operators; however, the physical relevance of such activity is rather questionable. The situation changes drastically when interaction is switched on. As beautifully explained by Weinberg [38-41] the most convenient (unique?) way of imposing the Lorentz covariance is to construct Lorentz covariant local fields with the help of Fourier transform supplied by relevant intertwining operators; such fields provide building blocks for constructing covariant interactions. The four coordinates which appear in this way should be considered as parametrizing the Minkowski space-time points. Operationally, they can be defined as positions of some heavy particles; the heavier they are the more unique is the notion of their localizability. The upper bound on their masses is related to the possibility of black hole formation which leads to the well known conclusion that the very notion of continuous space-time breaks at Planck length. In this way we get some feeling concerning the meaning of space-time coordinates. However, the question what is the relation between space-time coordinates and the coordinates of actual particles described by the field under consideration remains unsettled. In the case the interaction is present this problem becomes important. In both nonrelativistic or classical limits our understanding of localizability is quite complete. Moreover, in the weak field regime where particle number is approximately conserved we can look for the effective one-particle dynamics which should be formulated in terms of coordinate and momenta.

The quasiclassical theory of Weyl fermions provides a good laboratory for studying the relation between the dynamical variables characterizing a particle and the corresponding wave function/field. In particular, this concerns the transformation properties of both objects. We are dealing with one-particle approximation to relativistic interacting theory and ask for the transformation rules of the particle dynamical variables following from the 
transformation properties of the relevant wave function. The transformation rules are derived from first principles because the dynamical variables entering the action are precisely defined. In spite of their form (cf. eq. (2.15)) they are not obtained as a simple extension of nonrelativistic variables but emerge naturally from the relevant variational principle in semiclassical approximation. For example, eq. (2.15) is only valid provided pair creation is neglected. Once the precise definition of dynamical variables in the approximation under consideration is given, their relativistic transformation rules follow from the covariance of Weyl equation (in particular, from usual transformation rules of space-time arguments of fields entering Weyl equation). The advantage of such an approach over the one based on path integral stems from clear interpretation of particle coordinates which allows to replace an educated guess by a straightforward derivation of Lorentz transformation properties.

The action $I$ (eq. (1.1)) has been derived in [18] from that for Weyl fermions using the path integral method. However,as it has been pointed out above, in order to provide a clear interpretation of the dynamical variables entering (1.1) we prefer to use the method popular among condensed matter physicists [42]-[45] which is based on time-dependent variational principle.

The paper is organized as follows. In section 2 we consider free Weyl theory. Interaction with external electromagnetic field is considered in section 3. Some conclusions are presented in section 4. Appendix is devoted to some technicalities.

\section{Free Weyl fermions}

Let us start with free massless left-handed Weyl fermions. The relevant wave function obeys Weyl equation

$$
\sigma^{\mu} \partial_{\mu} \phi=0
$$

with $\sigma^{\mu}=(\mathbb{I},-\vec{\sigma}), \vec{\sigma}=\left(\sigma_{1}, \sigma_{2}, \sigma_{3}\right)$ being Pauli matrices. Eq. (2.1) is invariant under the action of Poincare group given by

$$
\phi_{\sigma}^{\prime}(x)=\left(A^{\dagger}\right)_{\sigma \sigma^{\prime}} \phi_{\sigma^{\prime}}(\Lambda x+a)
$$

where $\mathrm{SL}(2, \mathbb{C}) \ni A \longmapsto \Lambda(A) \in \mathrm{SO}(1,3)$ defines double (universal) covering of the Lorentz group. We shall consider the single particle theory described by positive energy solutions to eq. (2.1). They read

$$
\phi(\vec{x}, t)=\frac{1}{(2 \pi)^{\frac{3}{2}}} \int d^{3} \vec{p} c(\vec{p}) u_{+}(\vec{p}) e^{i(\vec{p} \vec{x}-|\vec{p}| t)},
$$

where $u_{+}(\vec{p})$ is the positive energy spinor obeying

$$
\vec{p} \cdot \vec{\sigma} u_{+}(\vec{p})=-|\vec{p}| u_{+}(\vec{p})
$$

together with the normalization condition

$$
u_{+}^{\dagger}(\vec{p}) u_{+}(\vec{p})=1
$$


The above normalization condition is not Lorentz invariant. On the other hand it is convenient because such a combination of spinors enters Lorentz invariant definition of scalar product ( being the space integral of zeroth component of current) and action functional $I$, eq. (2.10) below (resulting from invariant action functional for Dirac particle in the limit $m \rightarrow 0$ with one chirality deleted). Another choice of normalization would only influence the actual form of wave packet profile $c(\vec{p})$; the normalization condition enters then explicitly, for example, the expression for coordinate. However, all our conclusions remain valid.

Explicitly, $u_{+}(\vec{p})$ can be chosen as

$$
u_{+}(\vec{p})=\frac{1}{\sqrt{2|\vec{p}|\left(|\vec{p}|+p^{3}\right)}}\left(\begin{array}{c}
p^{1}-i p^{2} \\
-\left(|\vec{p}|+p^{3}\right)
\end{array}\right) .
$$

The wave function $\phi(\vec{x}, t)$, given by eq. (2.3), carries mass zero helicity $-\frac{1}{2}$ irreducible representation of Poincare group; $u_{+}(\vec{p})$ is the corresponding intertwining operator [38-41]. In fact, the generators of the representation (2.2) read

$$
\begin{gathered}
M_{\mu \nu}=i\left(x_{\mu} \partial_{\nu}-x_{\nu} \partial_{\mu}\right)+m_{\mu \nu}, \\
m_{i j}=\epsilon_{i j k} \frac{\sigma_{k}}{2}, \quad m_{0 k}=\frac{i \sigma_{k}}{2} .
\end{gathered}
$$

Their momentum counterparts, $\tilde{M}_{\mu \nu}$, acting in the space of momentum amplitudes, are given by

$$
\left(M_{\mu \nu} \phi\right)(\vec{x}, t)=\frac{1}{(2 \pi)^{\frac{3}{2}}} \int d^{3} \vec{p}\left(\tilde{M}_{\mu \nu} c\right)(\vec{p}) u_{+}(\vec{p}) e^{i(\vec{p} \vec{x}-|\vec{p}| t)}
$$

It is straightforward to check that $\tilde{M}_{\mu \nu}$ take the standard form of generators of massless helicity $-\frac{1}{2}$ representation of Poincare group (actually, its Lorentz subgroup). For example, the boosts read

$$
\begin{aligned}
\tilde{M}_{01} & =i|\vec{p}| \frac{\partial}{\partial p^{1}}+\frac{i p^{1}}{2|\vec{p}|}-\frac{p^{2}}{2\left(|\vec{p}|+p^{3}\right)} \\
\tilde{M}_{02} & =i|\vec{p}| \frac{\partial}{\partial p^{2}}+\frac{i p^{2}}{2|\vec{p}|}+\frac{p^{1}}{2\left(|\vec{p}|+p^{3}\right)}, \\
\tilde{M}_{03} & =i|\vec{p}| \frac{\partial}{\partial p^{3}}+\frac{i p^{3}}{2|\vec{p}|} .
\end{aligned}
$$

Eqs. (2.9) coincide with the well-known expressions (see, for example, [25, 46]) provided one takes into account that the invariant scalar product is defined here as $\int d^{3} \vec{p} \bar{c}_{1}(p) c_{2}(p)$ while usually the explicitly invariant measure $\frac{d^{3} \vec{p}}{2|\vec{p}|}$ is used. Note that eqs. (2.9) are not explicitly covariant under spatial rotations; this is because any choice of standard vector for massless representation of Poincare group necessarily breaks explicit rotational covariance. Let us also remind that eqs. (2.9) can be obtained by a straightforward quantization of the Hamiltonian system invariant under the action of the Poincare group, constructed with the help of coadjoint orbit method [25]. 
Up to now the information contained in eq. (2.1) (together with the choice of solutions with definite energy sign) is equivalent to that provided by the relevant unitary representation of Poincare group. Therefore, in principle, we do not have to consider space coordinates which are formally introduced by Fourier transform (cf. eq. (2.3)). However, they become important if we are going to describe interactions obeying locality principle necessary to provide relativistic invariance of interacting theory [38-41]. The space-time coordinates are commuting c-numbers transforming in the standard way; no exotic terms appear in Lorentz transformation rules. Therefore, the particle coordinates entering the semiclassical action must differ from space coordinates appearing in Weyl equation. In order to find the relation between them we derive the semiclassical action functional following the method based on time-dependent variational principle [42]-[45]. It provides a clear interpretation of the dynamical variables entering the action functional. To this end note that the action functional for Weyl fermions,

$$
I=i \int d^{4} x \phi^{\dagger} \sigma^{\mu} \partial_{\mu} \phi
$$

can be written in the form

$$
I=\int d t d^{3} \vec{x} \phi^{\dagger}(\vec{x}, t)\left(i \frac{\partial}{\partial t}-i \sigma_{k} \frac{\partial}{\partial x^{k}}\right) \phi(\vec{x}, t)=\int d t \mathcal{L}(t) .
$$

In order to find the effective Lagrangian $\mathcal{L}(t)$ we put

$$
\phi(\vec{x}, t)=\frac{1}{(2 \pi)^{\frac{3}{2}}} \int d^{3} \vec{p} c(\vec{p}, t) u_{+}(\vec{p}) e^{i \vec{p} \vec{x}},
$$

where $c(\vec{p}, t)$ is a wave packet profile. In the spirit of semiclassical approach we assume that $|c(\vec{p}, t)|$ is strongly peaked at some $\vec{p}_{c}(t)$. With this assumption $\mathcal{L}(t)$ can be easily computed from (2.11) and (2.12)

$$
\mathcal{L}(t)=\int d^{3} \vec{x} \phi^{+}(\vec{x}, t)\left(i \frac{\partial}{\partial t}-i \sigma_{k} \frac{\partial}{\partial x^{k}}\right) \phi(\vec{x}, t)=\frac{\partial \lambda\left(\vec{p}_{c}, t\right)}{\partial t}-\left|\vec{p}_{c}\right|
$$

where $\lambda(\vec{p}, t)$ is the phase function defined by

$$
c(\vec{p}, t)=|c(\vec{p}, t)| e^{-i \lambda(\vec{p}, t)} .
$$

The first term of the right-hand side of eq. (2.13) calls for more transparent interpretation. To this end we define the "center of charge" $\vec{x}_{c}$ by

$$
\vec{x}_{c}=\int d^{3} \vec{x} \phi^{\dagger}(\vec{x}, t) \phi(\vec{x}, t) \vec{x},
$$

(assuming $\phi(\vec{x}, t)$ is normalized to unity). With our assumption concerning $|c(\vec{p}, t)|$ eq. (2.15) yields

$$
\vec{x}_{c}=i u_{+}^{\dagger}\left(\vec{p}_{c}\right) \vec{\nabla}_{p_{c}} u_{+}\left(\vec{p}_{c}\right)+\vec{\nabla}_{p_{c}} \lambda\left(\vec{p}_{c}, t\right) .
$$

Combining eq. (2.13) and (2.16) one finds

$$
\mathcal{L}(t)=\vec{p}_{c} \dot{\vec{x}}_{c}-\left|\vec{p}_{c}\right|-\vec{\alpha}\left(\vec{p}_{c}\right) \dot{\vec{p}}_{c}+\frac{d}{d t}\left(\lambda\left(\vec{p}_{c}, t\right)-\vec{x}_{c} \vec{p}_{c}\right) \simeq \vec{p}_{c} \dot{\vec{x}}_{c}-\left|\vec{p}_{c}\right|-\vec{\alpha}\left(\vec{p}_{c}\right) \dot{\vec{p}}_{c}
$$


where

$$
\vec{\alpha}(\vec{p})=-i u_{+}^{\dagger}(\vec{p}) \vec{\nabla}_{p} u_{+}(\vec{p}) .
$$

We see that the derivation based on time-dependent variational principle yields indeed a transparent interpretation of dynamical variables entering the effective Lagrangian. Eq. (2.15) allows us to determine the transformation properties of $\vec{x}_{c}$. Consider, for example, an infinitesimal boost described by $\vec{\omega} \equiv\left(\omega^{01}, \omega^{02}, \omega^{03}\right)$. Eqs. (2.2) and (2.15), together with our assumption concerning the semiclassical character of the wave packet, yield the following transformation rule for $\vec{x}_{c}$ (valid on-shell, i.e. provided the Weyl equation (2.1) holds, cf. [18])

$$
\delta_{0} \vec{x}_{c}=t \vec{\omega}-\left(\vec{x}_{c} \cdot \vec{\omega}\right) \dot{\vec{x}}_{c}-\frac{\vec{\omega} \times \vec{p}_{c}}{2\left|\vec{p}_{c}\right|^{2}}+\left(\vec{x}_{c} \cdot \vec{\omega}\right)\left(\dot{\vec{x}}_{c}-\frac{\vec{p}_{c}}{\left|\vec{p}_{c}\right|}\right)
$$

The first two terms on the right-hand side refer to the standard Lorentz transformation rule (the second term accounts for the change of time variable); the third term is the exotic correction proposed in ref. [18]. Finally, the last term vanishes on semiclassical on-shell. Let us compare eq. (2.19) with the trajectory $\vec{x}=\vec{x}(t)$ of classical point particle. Under Lorentz transformation $\vec{x}^{\prime}\left(t^{\prime}\right)=\vec{x}(t)+t \vec{\omega}, t^{\prime}=t+\vec{\omega} \vec{x}$ yielding $\vec{x}^{\prime}(t)-\vec{x}(t)=t \vec{\omega}-(\vec{\omega} \vec{x}) \dot{\vec{x}}$. This shows that the third term on the right-hand side provides the additional helicitydependent contribution.

In order to find the interpretation of eq. (2.19) in the framework of unitary representation of Poincare group let us consider the question if there exists the coordinate operator corresponding to $\vec{x}_{c}$. One is tempted to define

$$
\left(\hat{\vec{x}}_{c} \phi\right)(\vec{x}, t)=\vec{x} \phi(\vec{x}, t)=\frac{1}{(2 \pi)^{\frac{3}{2}}} \int d^{3} \vec{p} i \vec{\nabla}_{p}\left(c(\vec{p}, t) u_{+}(\vec{p})\right) e^{i \vec{p} \vec{x}} .
$$

This is, however, wrong because $\vec{\nabla}_{p}\left(c(\vec{p}, t) u_{+}(\vec{p})\right)$ is not proportional to $u_{+}(\vec{p})$; in other words, $i \vec{\nabla}_{p}$ is not a one-particle operator. The cure for this is well-known: as in the case of Newton-Wigner operator [28] one has to project on positive energy states. Let us define the projector

$$
\Pi_{+}(\vec{p})=u_{+}(\vec{p}) u_{+}^{\dagger}(\vec{p})=\frac{1}{2}\left(\mathbb{I}-\frac{\vec{p} \vec{\sigma}}{|\vec{p}|}\right)
$$

The particle coordinate operator is now defined as

$$
\begin{aligned}
\left(\hat{\vec{x}}_{c} \phi\right)(\vec{x}, t) & =\frac{1}{(2 \pi)^{\frac{3}{2}}} \int d^{3} \vec{p} \Pi_{+}(\vec{p}) i \vec{\nabla}_{p} \Pi_{+}(\vec{p})\left(c(\vec{p}, t) u_{+}(\vec{p})\right) e^{i \vec{p} \vec{x}} \\
& =\frac{1}{(2 \pi)^{\frac{3}{2}}} \int d^{3} \vec{p} \Pi_{+}(\vec{p}) i \vec{\nabla}_{p}\left(c(\vec{p}, t) u_{+}(\vec{p})\right) e^{i \vec{p} \vec{x}} .
\end{aligned}
$$

Obviously, $\hat{\vec{x}}_{c}$ obeys

$$
\vec{x}_{c}=\int d^{3} \vec{x} \phi^{\dagger}(\vec{x}, t) \hat{\vec{x}}_{c} \phi(\vec{x}, t)
$$


It is straightforward to define the explicit expression for coordinates operator in momentum space:

$$
\begin{aligned}
& \hat{x}_{c}^{1}=i \frac{\partial}{\partial p^{1}}-\frac{p^{2}}{2|\vec{p}|\left(|\vec{p}|+p^{3}\right)}, \\
& \hat{x}_{c}^{2}=i \frac{\partial}{\partial p^{2}}+\frac{p^{1}}{2|\vec{p}|\left(|\vec{p}|+p^{3}\right)}, \\
& \hat{x}_{c}^{3}=i \frac{\partial}{\partial p^{3}} .
\end{aligned}
$$

It coincides (modulo the definition of scalar product) with the one given in [14-17, 25, 27]. The components of $\hat{\vec{x}}_{c}$ obey the commutation rules

$$
\left[\hat{x}_{c}^{k}, \hat{x}_{c}^{l}\right]=\frac{i}{2} \epsilon_{k l m} \frac{p^{m}}{|\vec{p}|^{3}} .
$$

It is not difficult to understand the origin of eq. (2.25). As it has been already mentioned the standard vector defining massless representation cannot be chosen in rotationally invariant way. As a consequence, for some variables the rotational covariance is implemented in a form of nonlinear realization of $\mathrm{SU}(2)$ linearizing on some subgroup $\mathrm{U}(1)$. As far as coordinate variables are concerned we are left with the following alternative: either we choose the coordinates transforming in nonstandard way under rotations or they form an usual three-vector but fail to obey canonical commutation rules [25]. Let us note that in the presence of external fields the commutation rule (2.25) must be generalized. However, the transformation rules for dynamical variables, both in the free (eq. (2.19)) and interacting (eqs. (3.10) and (3.12)) cases can be derived without using general commutation rules so there is no point to quote here the latter.

Passing on shell, $c(\vec{p}, t)=c(\vec{p}) \exp (-i|\vec{p}| t)$ one finds the form of $\vec{x}_{c}$ in Heisenberg picture (acting on $c(\vec{p}))$ :

$$
\hat{\vec{x}}_{c}(t)=\hat{\vec{x}}_{c}+t \frac{\vec{p}}{|\vec{p}|} .
$$

Poincare generators may be expressed in terms of $\vec{x}_{c}$ and $\vec{p}$. For example, the boosts read (cf. eqs. (2.9) and (2.24)):

$$
\tilde{M}_{0 k}=\frac{1}{2}\left(|\vec{p}| \hat{x}_{c}^{k}+\hat{x}_{c}^{k}|\vec{p}|\right)
$$

or

$$
\tilde{M}_{0 k}=\frac{1}{2}\left(|\vec{p}| \hat{x}_{c}^{k}(t)+\hat{x}_{c}^{k}(t)|\vec{p}|\right)-p^{k} t \equiv \tilde{M}_{0 k}(t)-p^{k} t .
$$

In order to find the transformation property of $\vec{x}_{c}(t)$ we compute

$$
\left[\hat{x}_{c}^{l}(t), \tilde{M}_{0 k}\right]=\left[\hat{x}_{c}^{l}(t), \tilde{M}_{0 k}(t)-t p^{k}\right]=\frac{i}{2}\left(\frac{p^{l}}{|\vec{p}|} \hat{x}_{c}^{k}(t)+\hat{x}_{c}^{k}(t) \frac{p^{l}}{|\vec{p}|}\right)-i t \delta_{l k}+\frac{i}{2} \epsilon_{l k m} \frac{p^{m}}{|\vec{p}|^{2}}
$$

For infinitesimal Lorentz transformation one has

$$
\delta \hat{\vec{x}}_{c}(t)=\left[\hat{\vec{x}}_{c}(t), i \omega^{0 k} M_{0 k}\right]
$$

Eqs. (2.23), (2.29) and (2.30), together with the assumption concerning the shape of wave packet, imply again the transformation rule (2.19). 


\section{$3 \quad$ Interaction with electromagnetic field}

Let us now consider the Weyl fermions interacting with external electromagnetic field. The Weyl equation reads now

$$
\sigma^{\mu}\left(\partial_{\mu}+i A_{\mu}\right) \phi=0
$$

which corresponds to the Hamiltonian

$$
H=\vec{\sigma}\left(i \frac{\partial}{\partial \vec{x}}+\vec{A}\right)+A^{0}, \quad \vec{A}=\left(A^{1}, A^{2}, A^{3}\right) .
$$

In order to derive the semiclassical action we follow closely the method presented in [43]. To this end we consider the wave packet centered at $\vec{x}_{c}$ at a given time with its spread small as compared to the length scale of variability of electromagnetic field. The local Hamiltonian (in the terminology of [43]) reads

$$
\hat{H}_{c}=\vec{\sigma}\left(i \frac{\partial}{\partial \vec{x}}+\vec{A}\left(\vec{x}_{c}, t\right)\right)+A^{0}\left(\vec{x}_{c}, t\right) .
$$

The solution to local eigenvalue equation

$$
\hat{H}_{c} \phi_{p}(\vec{x}, t)=E_{c_{p}} \phi_{p}(\vec{x}, t)
$$

has the form

$$
\begin{aligned}
\phi_{p}(\vec{x}, t) & =u_{+}\left(\vec{p}-A\left(\vec{x}_{c}, t\right)\right) e^{i \vec{p} \vec{x}}, \\
E_{c_{p}} & =\left|\vec{p}-\vec{A}\left(\vec{x}_{c}, t\right)\right|+A^{0}\left(\vec{x}_{c}, t\right),
\end{aligned}
$$

where $u_{+}\left(\vec{p}-\vec{A}\left(\vec{x}_{c}, t\right)\right)$ obeys

$$
\vec{\sigma}\left(\vec{p}-\vec{A}\left(\vec{x}_{c}, t\right)\right) u_{+}=-\left|\vec{p}-\vec{A}\left(\vec{x}_{c}, t\right)\right| u_{+} .
$$

The solution (3.5) has been chosen as the one which evolves adiabatically from positive energy solution to free Weyl equation; this choice is dictated by the assumption that we are working in the framework of single-particle theory.

Consider now the wave packet

$$
\phi(\vec{x}, t)=\frac{1}{(2 \pi)^{\frac{3}{2}}} \int d^{3} \vec{p} c(\vec{p}, t) u_{+}\left(\vec{p}-\vec{A}\left(\vec{x}_{c}, t\right)\right) e^{i \vec{p} \vec{x}},
$$

and assume again that $c(\vec{p}, t)$ is strongly peaked at some $\vec{p}_{c}(t)$. The consistency condition is that the above wave packet yields the pre-assigned center position which implies that eq. (2.15) is still valid. The starting point for derivation of effective semiclassical action is again the time-dependent variational principle for the action functional

$$
I=\int d t \int d^{3} \vec{x} \phi^{\dagger}(\vec{x}, t)\left(i \frac{\partial}{\partial t}-H\right) \phi(\vec{x}, t)
$$


with $H$ an $\phi$ given by eqs. (3.2) and (3.7), respectively. Following the steps described in ref. [43] (which involve, in particular, Taylor expansion of potentials to first order) we find after slightly troublesome calculations

$$
I=\int d t\left(\left(\vec{q}_{c}+\vec{A}\left(\vec{x}_{c}, t\right)\right) \dot{\vec{x}}_{c}-\left(\left|\vec{q}_{c}\right|+A^{0}\left(\vec{x}_{c}, t\right)+\frac{\vec{q}_{c} \cdot \vec{B}\left(\vec{x}_{c}, t\right)}{2\left|\vec{q}_{c}\right|^{2}}\right)-\vec{\alpha}\left(\vec{q}_{c}\right) \cdot \dot{\vec{q}}_{c}\right)
$$

which agrees with previous findings; here $\vec{q}_{c} \equiv \vec{p}_{c}-\vec{A}\left(\vec{x}_{c}, t\right)$ is the gauge-invariant kinetic momentum. Let us stress again that the wave packet method provides a clear interpretation of the variables entering action functional. In particular, $\vec{x}_{c}$ is again given by (2.15). Its transformation properties can be derived from eqs. (2.2) and (2.15). In particular, the Lorentz boosts take the form

$$
\delta_{0} x_{c}^{i}=\omega^{0 i} t-\omega^{0 k} x_{c}^{k} \dot{x}_{c}^{i}+\omega^{0 k} x_{c}^{k}\left(\dot{x}_{c}^{i}-\frac{q_{c}^{i}}{\left|\vec{q}_{c}\right|}\right)+\frac{\left(\vec{q}_{c} \times \vec{\omega}\right)^{i}}{2\left|\vec{q}_{c}\right|^{2}}
$$

(let us remind that $\left.\vec{\omega}=\left(\omega^{01}, \omega^{02}, \omega^{03}\right)\right)$.

The first term on the right-hand side is the standard Lorentz transformation rule for the spatial part of $x^{\mu}$; the second one accounts for the time variation $\left(\delta t=\omega^{0 k} x^{k}\right)$ since we are working in fixed time (Hamiltonian) formalism while the third term vanishes onshell. Therefore, our result agrees with previous findings [14-18, 22-24]. It is important to note that the on-shell condition is defined using the equations of motion to zeroth order in Planck constant, in spite of the fact that we are considering the transformation properties to the first order in $\hbar$. This is because the variation of the action (3.9) under Lorentz transformations to the order $\hbar^{1}$ is proportional (up to boundary terms) to the EulerLagrange expressions to the order $\hbar^{0}$ (cf. ref. [18]) and one can use the equations of motion to this order to account for time variation (see appendix). Similar reasoning (although a little bit more involved) gives the transformation rule for the momentum variable. Let us note that

$$
\vec{p}_{c}=\int d^{3} \vec{p}|c(\vec{p}, t)|^{2} \vec{p}=\int d^{3} \vec{x} \phi^{\dagger}(\vec{x}, t)(-i \vec{\nabla}) \phi(\vec{x}, t) .
$$

In the noninteracting case eq. (3.11) implies standard transformation rule for $\vec{p}_{c}$. For nonvanishing external fields things get more complicated. The transformation rule for $\vec{p}_{c}$ involves fields; moreover, we are interested in transformation properties of gauge-invariant kinetic momentum $\vec{q}=\vec{p}-\vec{A}$ rather than those of canonical momentum $\vec{p}$. After slightly tedious but straightforward calculations one finds

$$
\begin{aligned}
\delta q_{c}^{i}= & \omega^{0 i}\left(\left|\vec{q}_{c}\right|+\frac{\vec{q}_{c} \cdot \vec{B}}{2\left|\vec{q}_{c}\right|^{2}}\right)+\frac{\left(\left(\vec{q}_{c} \times \vec{\omega}\right) \times \vec{B}\right)^{i}}{2\left|\vec{q}_{c}\right|^{2}}+\left(\vec{\omega} \cdot \vec{x}_{c}\right)\left[\left(\dot{\vec{x}}_{c}-\frac{\vec{q}_{c}}{\left|\vec{q}_{c}\right|}\right) \times \vec{B}\right]^{i} \\
& +\left(\omega \cdot \vec{x}_{c}\right)\left[\dot{\vec{q}}_{c}-\vec{E}-\left(\dot{\vec{x}}_{c} \times \vec{B}\right)\right]^{i} .
\end{aligned}
$$

To derive eq. (3.12) we have used the transformation property of external potentials, $A^{\prime \mu}\left(x^{\prime}\right)=\Lambda_{\nu}^{\mu} A^{\nu}(x), x^{\prime \mu}=\Lambda^{\mu}{ }_{\nu} x^{\nu}$, corrected by taking into account the last terms on the right-hand of eq. (3.10). Using equations of motion (again to the zeroth order in $\hbar$, cf. the remark after eq. (3.10)) one checks that eq. (3.12) agrees with the previous findings. 


\section{Conclusions}

We have considered the dynamics of massless chiral fermions in semiclassical approximation, both for free fermions and fermions interacting with background electromagnetic field. Assuming that the momentum profile of the wave packet is strongly peaked at some value $\vec{p}_{c}$ while its space spread is small as compared to the length scale of variability of external field, we were able to derive the effective action for single particle sector (i.e. neglecting pair creation). To this end we used time-dependent variational principle which allows for transparent interpretation of canonical variables $\vec{x}_{c}$ and $\vec{p}_{c}$. In particular, $\vec{x}_{c}$ defines the position of the center of charge of the wave packet (cf. eq. (2.15)). However, even in the free case the commuting space variables $\vec{x}$ entering the Weyl equation and indexing the space points, cannot be considered as the eigenvalues of coordinate operator defined in the framework of single-particle theory. On the contrary, the properly defined coordinate operator $\hat{\vec{x}}_{c}$ has noncommuting components (cf. eq. (2.25)) which cannot be diagonalized simultaneously (they rather have to obey $\Delta x_{c}^{i} \Delta x_{c}^{j} \geq \frac{1}{4} \mid \frac{\left|p_{c}^{k}\right|}{\left|\vec{p}_{c}\right|^{3}}$, with $(i j k)$ being a cyclic permutation). The relation between space-time coordinates and the coordinate operator becomes even more complicated in the case of fermions interacting with background electromagnetic field. Once again $\hat{\vec{x}}_{c}$ is defined by equations analogous to eqs. (2.20)-(2.22); however, such a definition involves implicitly the actual value of $\vec{x}_{c}$.

In any case, the above mentioned relation appears to be well defined in the framework of time-dependent variational principle. This allows us to derive the proper transformation rules for particle coordinates and momenta from their very definitions showing the covariance of massless chiral theory (to the first order in $\hbar$ ). What is more, it provides a deeper insight into the problem of how the space-time arguments entering relativistic wave functions/fields should be interpreted. In particular, it appears that the relation between space-time and particle coordinates depends on the context and the approximation used; the main point is that the interaction should be weak enough to justify neglecting pair creation. Then one can work within one particle approximation and the attributes of single particle acquire well defined meaning.

Finally, let us note that the Weyl equation enjoys larger symmetry group - the conformal one. In fact, an unitary representation of conformal group corresponding to massless particles can be desribed in terms of operators from envelopping algebra of Poincare algebra. Therefore, the results obtained above can be extended to include the scaling and special conformal transformations. Details will be presented elsewhere.

\section{Acknowledgments}

This work has been supported by the grant 2016/23/B/ST2/00727 of National Science Centre, Poland.

\section{A Variation of action to the first order in $\hbar$}

In order to show that one can use the equations of motion to zeroth order in $\hbar$ to account for the time variation, let us consider the total variation of $\vec{x}_{c}$ and $\vec{q}_{c}$ obtained by taking 
into account the full (i.e. as to first order in $\hbar$ ) equations of motion. Then eqs. (3.10) and (3.12) are replaced by

$$
\delta x_{c}^{i}=\omega^{0 i} t+\frac{\left(\vec{q}_{c} \times \vec{\omega}\right)^{i}}{2\left|\vec{q}_{c}\right|^{2}}+\vec{\omega} \cdot \vec{x}_{c}\left(\vec{\nabla}_{q_{c}}\left(\frac{\vec{q}_{c} \cdot \vec{B}}{2\left|\vec{q}_{c}\right|^{2}}\right)+\frac{\left(\vec{q}_{c} \times \dot{\vec{q}}_{c}\right)}{2\left|\vec{q}_{c}\right|^{3}}\right)^{i},
$$

and

$$
\begin{aligned}
\delta q_{c}^{i}= & \omega^{0 i}\left(\left|\vec{q}_{c}\right|+\frac{\vec{q}_{c} \cdot \vec{B}}{2\left|\vec{q}_{c}\right|^{2}}\right)+\frac{\left(\left(\vec{q}_{c} \times \vec{\omega}\right) \times \vec{B}\right)^{i}}{2\left|\vec{q}_{c}\right|^{2}} \\
& +\left(\vec{\omega} \cdot \vec{x}_{c}\right)\left[\left(\vec{\nabla}_{q_{c}}\left(\frac{\vec{q}_{c} \cdot \vec{B}}{2\left|\vec{q}_{c}\right|^{2}}\right)+\frac{\left(\vec{q}_{c} \times \dot{\dot{q}_{c}}\right)}{2\left|\vec{q}_{c}\right|^{3}}\right) \times \vec{B}\right]^{i}-\left(\vec{\omega} \cdot \vec{x}_{c}\right)\left(\vec{\nabla}_{x}\left(\frac{\vec{q}_{c} \cdot \vec{B}}{2\left|\vec{q}_{c}\right|^{2}}\right)\right)^{i},
\end{aligned}
$$

respectively. Calling $I_{0}$ and $I_{1}$ the $O\left(\hbar^{0}\right)$ and $O\left(\hbar^{1}\right)$ parts of the action (3.9) we have to show that $\delta I_{0}$ vanishes up to boundary terms provided it is computed by taking into account only additional terms appearing on the right-hand sides of eqs. (A.1) and (A.2). Computing the relevant variation (cf. ref. [18]) one obtains

$$
\begin{aligned}
\delta I_{0}= & \int d t\left[\left(\vec{\omega} \cdot \vec{x}_{c}\right)\left(\vec{\nabla}_{q_{c}}\left(\frac{\vec{q}_{c} \cdot \vec{B}}{2\left|\vec{q}_{c}\right|^{2}}\right)+\frac{\left(\vec{q}_{c} \times \dot{\vec{q}}_{c}\right)}{2\left|\vec{q}_{c}\right|^{3}}\right) \cdot\left(-\dot{\vec{q}}_{c}+\vec{E}+\dot{\vec{x}}_{c} \times \vec{B}\right)\right. \\
& +\left(\vec{\omega} \cdot \vec{x}_{c}\right) \cdot\left(\left(\vec{\nabla}_{q_{c}}\left(\frac{\vec{q}_{c} \cdot \vec{B}}{2\left|\vec{q}_{c}\right|^{2}}\right)+\frac{\left(\vec{q}_{c} \times \dot{\vec{q}}_{c}\right)}{2\left|\vec{q}_{c}\right|^{3}}\right) \times \vec{B}\right)\left(\dot{\vec{x}}_{c}-\frac{\vec{q}_{c}}{\left|\vec{q}_{c}\right|}\right) \\
& \left.-\left(\vec{\omega} \cdot \vec{x}_{c}\right)\left(\dot{\vec{x}}_{c}-\frac{\vec{q}_{c}}{\left|\vec{q}_{c}\right|}\right)\left(\vec{\nabla}_{x}\left(\frac{\vec{q}_{c} \cdot \vec{B}}{2\left|\vec{q}_{c}\right|^{2}}\right)\right)+\frac{d(\ldots)}{d t}\right]
\end{aligned}
$$

which reduces to the total derivative on-shell (defined by full equations of motion).

Open Access. This article is distributed under the terms of the Creative Commons Attribution License (CC-BY 4.0), which permits any use, distribution and reproduction in any medium, provided the original author(s) and source are credited.

\section{References}

[1] D.T. Son and P. Surówka, Hydrodynamics with Triangle Anomalies, Phys. Rev. Lett. 103 (2009) 191601 [arXiv:0906.5044] [INSPIRE].

[2] R. Loganayagam, Anomaly Induced Transport in Arbitrary Dimensions, arXiv:1106.0277 [INSPIRE].

[3] R. Loganayagam and P. Sur owka, Anomaly/Transport in an Ideal Weyl gas, JHEP 04 (2012) 097 [arXiv:1201.2812] [INSPIRE].

[4] S. Dubovsky, L. Hui, A. Nicolis and D.T. Son, Effective field theory for hydrodynamics: thermodynamics and the derivative expansion, Phys. Rev. D 85 (2012) 085029 [arXiv: 1107.0731] [INSPIRE]. 
[5] S. Dubovsky, L. Hui and A. Nicolis, Effective field theory for hydrodynamics: Wess-Zumino term and anomalies in two spacetime dimensions, Phys. Rev. D 89 (2014) 045016.

[6] K. Fukushima, D.E. Kharzeev and H.J. Warringa, The Chiral Magnetic Effect, Phys. Rev. D 78 (2008) 074033 [arXiv:0808.3382] [INSPIRE].

[7] K. Fukushima, D.E. Kharzeev and H.J. Warringa, Real-time dynamics of the Chiral Magnetic Effect, Phys. Rev. Lett. 104 (2010) 212001 [arXiv:1002.2495] [INSPIRE].

[8] G. Basar, G.V. Dunne and D.E. Kharzeev, Chiral Magnetic Spiral, Phys. Rev. Lett. 104 (2010) 232301 [arXiv:1003.3464] [INSPIRE].

[9] M.A. Stephanov and Y. Yin, Chiral Kinetic Theory, Phys. Rev. Lett. 109 (2012) 162001 [arXiv: 1207.0747] [INSPIRE].

[10] D.T. Son and N. Yamamoto, Berry Curvature, Triangle Anomalies and the Chiral Magnetic Effect in Fermi Liquids, Phys. Rev. Lett. 109 (2012) 181602 [arXiv:1203.2697] [INSPIRE].

[11] D.T. Son and N. Yamamoto, Kinetic theory with Berry curvature from quantum field theories, Phys. Rev. D 87 (2013) 085016.

[12] J.-W. Chen, S. Pu, Q. Wang and X.-N. Wang, Berry Curvature and Four-Dimensional Monopoles in the Relativistic Chiral Kinetic Equation, Phys. Rev. Lett. 110 (2013) 262301 [arXiv: 1210.8312] [INSPIRE].

[13] J.-W. Chen, J.-y. Pang, S. Pu and Q. Wang, Kinetic equations for massive Dirac fermions in electromagnetic field with non-Abelian Berry phase, Phys. Rev. D 89 (2014) 094003 [arXiv: 1312.2032] [INSPIRE].

[14] M. Stone and V. Dwivedi, Classical version of the non-Abelian gauge anomaly, Phys. Rev. D 88 (2013) 045012 [arXiv: 1305.1955] [INSPIRE].

[15] V. Dwivedi and M. Stone, Classical chiral kinetic theory and anomalies in even space-time dimensions, J. Phys. A 47 (2014) 025401.

[16] M. Stone, V. Dwivedi and T. Zhou, Berry Phase, Lorentz Covariance and Anomalous Velocity for Dirac and Weyl Particles, Phys. Rev. D 91 (2015) 025004 [arXiv:1406.0354] [INSPIRE].

[17] M. Stone, V. Dwivedi and T. Zhou, Wigner Translations and the Observer Dependence of the Position of Massless Spinning Particles, Phys. Rev. Lett. 114 (2015) 210402 [arXiv: 1501.04586] [INSPIRE].

[18] J.-Y. Chen, D.T. Son, M.A. Stephanov, H.-U. Yee and Y. Yin, Lorentz Invariance in Chiral Kinetic Theory, Phys. Rev. Lett. 113 (2014) 182302 [arXiv:1404.5963] [INSPIRE].

[19] E. Megias and M. Valle, Second-order partition function of a non-interacting chiral fluid in 3+1 dimensions, JHEP 11 (2014) 005 [arXiv: 1408.0165] [INSPIRE].

[20] K.A. Sohrabi, Microscopic Study of Vorticities in Relativistic Chiral Fermions, JHEP 03 (2015) 014 [arXiv:1409.4528] [INSPIRE].

[21] C. Manuel and J.M. Torres-Rincon, Chiral transport equation from the quantum Dirac Hamiltonian and the on-shell effective field theory, Phys. Rev. D 90 (2014) 076007 [arXiv: 1404.6409] [INSPIRE].

[22] P. Zhang and P.A. Horváthy, Anomalous Hall Effect for semiclassical chiral fermions, Phys. Lett. A 379 (2014) 507 [arXiv:1409.4225] [inSPIRE]. 
[23] C. Duval and P.A. Horvathy, Chiral fermions as classical massless spinning particles, Phys. Rev. D 91 (2015) 045013 [arXiv: 1406.0718] [INSPIRE].

[24] C. Duval, M. Elbistan, P.A. Horváthy and P.M. Zhang, Wigner-Souriau translations and Lorentz symmetry of chiral fermions, Phys. Lett. B 742 (2015) 322 [arXiv:1411.6541] [INSPIRE].

[25] K. Andrzejewski, A. Kijanka-Dec, P. Kosiński and P. Maślanka, Chiral fermions, massless particles and Poincaré covariance, Phys. Lett. B 746 (2015) 417 [arXiv:1503.07101] [INSPIRE].

[26] J.-M. Souriau, Structure of Dynamical Systems: A Sympletic View of Physics, Birkhäuser, Boston U.S.A. (1997).

[27] B.S. Skagerstam, Localization of massless spinning particles and the Berry phase, hep-th/9210054 [INSPIRE].

[28] T.D. Newton and E.P. Wigner, Localized States for Elementary Systems, Rev. Mod. Phys. 21 (1949) 400 [INSPIRE].

[29] K. Yu. Bliokh and Yu. P. Bliokh, Topological spin transport of photons: The Optical Magnus Effect and Berry Phase, Phys. Lett. A 333 (2004) 181 [physics/0402110] [InSPIRE].

[30] M. Onoda, S. Murakami and N. Nagaosa, Hall Effect of Light, Phys. Rev. Lett. 93 (2004) 083901 [cond-mat/0405129] [INSPIRE].

[31] C. Duval, Z. Horvath and P. Horvathy, Geometrical spinoptics and the optical Hall effect, J. Geom. Phys. 57 (2007) 925 [math-ph/0509031] [InSPIRE].

[32] C. Duval, Z. Horvath and P.A. Horvathy, Fermat principle for spinning light, Phys. Rev. D 74 (2006) 021701 [cond-mat/0509636] [INSPIRE].

[33] K. Bliokh, A. Niv, V. Kleiner and E. Hasman, Geometrodynamics of Spinning Light, Nature Photon. 2 (2008) 748 [arXiv: 0810.2136].

[34] K. Bliokh, Geometrodynamics of polarized light: Berry phase and spin Hall effect in a gradient-index medium, J. Opt. A 11 (2009) 094009.

[35] K.Y. Bliokh and F. Nori, Relativistic Hall Effect, Phys. Rev. Lett. 108 (2012) 120403 [arXiv: 1112.5618] [INSPIRE].

[36] L. Landau and R. Peierls, Time of the Energy Emission in the Hydrogen Atom and Its Electrodynamical Background, Z. Phys. 69 (1931) 56.

[37] V.B. Berestetskii, E.M. Lifshitz and L.P. Pitaevskii, Relativistic Quantum Theory 1, Pergamon Press, Oxford U.K. (1971).

[38] S. Weinberg, Feynman Rules for Any Spin, Phys. Rev. 133 (1964) B1318 [InSPIRE].

[39] S. Weinberg, Feynman Rules for Any Spin. 2. Massless Particles, Phys. Rev. 134 (1964) B882 [INSPIRE].

[40] S. Weinberg, Photons and Gravitons in s Matrix Theory: Derivation of Charge Conservation and Equality of Gravitational and Inertial Mass, Phys. Rev. 135 (1964) B1049 [InSPIRE].

[41] S. Weinberg, The Quantum Theory of Fields. Vol. I, Cambridge University Press, Cambridge U.K. (1995).

[42] M.-C. Chang and Q. Niu, Berry phase, hyperorbits and the Hofstadter spectrum: Semiclassical dynamics in magnetic Bloch bands, Phys. Rev. B 53 (1996) 7010 [INSPIRE]. 
[43] G. Sundaram and Q. Niu, Wave-packet dynamics in slowly perturbed crystals: Gradient corrections and Berry-phase effects, Phys. Rev. B 59 (1999) 14915 [cond-mat/9908003] [INSPIRE].

[44] D. Culcer, Y. Yao and Q. Niu, Coherent wave-packet evolution in coupled bands, Phys. Rev. B 72 (2005) 085110.

[45] M.-C. Chang and Q. Niu, Berry curvature, orbital moment, and effective quantum theory of electrons in electromagnetic fields, J. Phys. Condens. Matter 20 (2008) 193202.

[46] Yu. V. Novozhilov, Introduction to Elementary Particle Theory, Pergamon Press, Oxford U.K. (1975). 